\title{
A REPRESENTATION THEOREM FOR BIEQUICONTINUOUS COMPLETED TENSOR PRODUCTS OF WEIGHTED SPACES $\left({ }^{1}\right)$
}

\author{
BY \\ W. H. SUMMERS
}

1. Introduction. Interest in weighted spaces of continuous functions dates back at least to the Bernstein approximation problem [1], while Nachbin [8], [9] has made more recent use of these spaces in approximation theory. Moreover, weighted spaces provide a general setting for the study of virtually all continuous function spaces encountered in analysis (leading to new results, as well as new, more elegant proofs of known results).

In this paper we will be mainly concerned with obtaining a representation of the biequicontinuous completed tensor product of two weighted spaces as another weighted space (via a topological isomorphism). Among its several corollaries, this theorem includes Grothendieck's result [6] for $C_{0}(X) \otimes \approx C_{0}(Y)$, establishes an analogous result for the space $\left(C_{b}(X), \beta\right)$ of bounded continuous functions with the strict topology (see Buck [2]), and leads to the discovery of some interesting new spaces of continuous functions on $X \times Y$.

The technique for obtaining this representation, however, entails an investigation of weighted spaces as locally convex spaces, and this leads to several results of independent interest. In particular, we are able to characterize the topological dual spaces of weighted spaces, and this characterization (which has the flavor of a factorization theorem) has several applications other than those considered here (see [10]).

2. Preliminaries. Throughout the remainder of this paper, $X$ (and $Y$ ) will denote a completely regular $T_{1}$-space (although we will find occasion to hypothesize additional and even stronger properties), while all functions will be complex valued unless it is explicitly stated otherwise. We shall let $C(X)$ denote the space of all continuous functions on $X, B(X)$ denote the space of all bounded functions on $X$, and $B_{0}(X)$ denote the space of all functions on $X$ which vanish at infinity. Further, let $C_{b}(X)=C(X) \cap B(X)$ and $C_{0}(X)=C(X) \cap B_{0}(X)$, while $C_{c}(X)$ will denote those continuous functions on $X$ which have compact support and $N(X)$ will

Received by the editors October 28, 1968 and, in revised form, June 17, 1969.

(') This article is taken primarily from the author's doctoral dissertation submitted to Louisiana State University in August, 1968. The author wishes to express his gratitude to Professor Heron S. Collins for his advice and encouragement.

Copyright (C) 1969, American Mathematical Society 
denote those functions $f$ on $X$ with the property that $|f|$ is upper semicontinuous (u.s.c.). If $f$ is a function on $X$, then $N(f)=\{x \in X: f(x) \neq 0\}$ and spt $(f)=N(f)^{-}$. Moreover, if $S \subseteq X$, then $R(f ; S)$ will denote the restriction of $f$ to $S$, and if $R(f ; S) \in B(S)$, then define $\|f\|_{s}=\sup \{|f(x)|: x \in S\}$. In the case $S=X$ we write $\|f\|$, and will refer to the topology induced by $\|\cdot\|$ on $B(X)$ as the uniform topology.

3. Weighted locally convex spaces of continuous functions. A Nachbin family $V$ on $X$ (compare Nachbin [8], [9]) is a set of nonnegative u.s.c. functions on $X$ satisfying the condition that if $u, v \in V$ and $\lambda \geqq 0$, then there is a $w \in V$ such that $\lambda u, \lambda v \leqq w$ (pointwise). If $V$ is a Nachbin family on $X$, then the corresponding weighted space $C V_{0}(X)=\left\{f \in C(X): f v \in B_{0}(X)\right.$ for every $\left.v \in V\right\}$ endowed with the weighted topology $\omega$ (or $\omega_{V}$ ) generated by the set of seminorms $\left\{p_{v}: v \in V\right\}$ where $p_{v}(f)=\|f v\|$ for each $f \in C V_{0}(X) . C V_{0}(X)$ is a locally convex space, and a base of closed, absolutely convex neighborhoods of zero is formed by the sets

$$
V_{v}=\left\{f \in C V_{0}(X):\|f v\| \leqq 1\right\}, \quad v \in V .
$$

Unless otherwise specified, we will consider only those Nachbin families $V$ on $X$ for which $C V_{0}(X)$ is Hausdorff. This is the case if and only if for each open $A \subseteq X$ for which there is an $f \in C V_{0}(X)$ such that $R(f ; A) \neq 0$, then there is a $v \in V$ such that $R(v ; A) \neq 0$. If $U$ and $V$ are two Nachbin families on $X$ and if for each $u \in U$ there is a $v \in V$ such that $u \leqq v$, then we write $U \leqq V$. In case $U \leqq V$ and $V \leqq U$, we write $U \approx V$.

3.1. THEOREM. If $U$ and $V$ are Nachbin families on $X$ with $U \leqq V$, then

(1) $C V_{0}(X) \subseteq C U_{0}(X)$, and

(2) $r\left(\omega_{U} ; C V_{0}(X)\right) \subseteq \omega_{V}$.

(In analogy with our symbol for the restriction of a function, $r(\mathscr{T} ; E)$ will denote the relative topology induced on the subset $E$ of a topological space $(F ; \mathscr{T})$ by the topology $\mathscr{T}$.)

Proof. This is an immediate consequence of the definitions.

3.2. Corollary. If $U$ and $V$ are Nachbin families on $X$ with $U \approx V$, then $C U_{0}(X)=C V_{0}(X) ;$ i.e., they are the same sets with the same topologies.

We have the following partial converse of 3.1.

3.3. THEOREM. Let $U$ and $V$ be Nachbin families on $X$ and assume (1) and (2) of 3.1 hold. If either

(i) $V \subseteq B_{0}^{+}(X)$ or (ii) $X$ is locally compact, then $U \leqq V$.

Proof. If $u \in U$, then there is a $v \in V$ such that $V_{v} \subseteq V_{u} \cap C V_{0}(X)$. If we set $A=\{x \in X:(u-v)(x)>0\}$, we would show $A$ is void. If not, let $x_{0} \in A$ and let $B=\left\{x \in X: v(x)<\frac{1}{2}\left(v\left(x_{0}\right)+u\left(x_{0}\right)\right)\right\}$. Then $B$ is open and $x_{0} \in B$, which implies there exists $\theta \in C(X)$ such that $0 \leqq \theta \leqq 1, \theta\left(x_{0}\right)=1$, and $\theta(X \mid B)=0$. If (ii) holds, 
then we may assume $\theta \in C_{c}(X)$, in which case it is clear that $\theta \in C V_{0}(X)$; while if (i) holds, then for $w \in V$ and $\varepsilon>0$ we have

$$
K=\{x \in X: \theta(x) w(x) \geqq \varepsilon\} \subseteq\{x \in X: w(x) \geqq \varepsilon\},
$$

and this implies $K$ is compact from whence it follows that $\theta \in C V_{0}(X)$.

So in either case, $f=2\left(v\left(x_{0}\right)+u\left(x_{0}\right)\right)^{-1} \theta$ is in $C V_{0}(X)$ and $|f(x)| v(x) \leqq 1$ for every $x \in X$, which implies $f \in V_{v}$. But $f\left(x_{0}\right) u\left(x_{0}\right)>1$, which contradicts $f \in V_{u}$. Hence $A$ is void and the proof is complete.

3.4. EXAMPLES. For a subset $S \subseteq X$, we will denote the characteristic function of $S$ by $\chi_{s}$. If $\chi_{c}(X)=\left\{\lambda \chi_{K}: \lambda \geqq 0, K \subseteq X, K\right.$ compact $\}$ and if $V=\chi_{c}(X)$, then $V$ is a Nachbin family on $X$ and $C V_{0}(X)$ is $C(X)$ endowed with the compact-open (c-op) topology. If $\mathscr{A}$ is any vector subspace of $C(X)$, then $\mathscr{A}^{+}$is a Nachbin family on $X$. Hence $U=C_{c}^{+}(X)$ is a Nachbin family on $X$, and it is an easy consequence of 3.1 and 3.3 that $X$ is locally compact if (and only if) $U \approx V$. Therefore $C U_{0}(X)$ is $(C(X)$, c-op) whenever $X$ is locally compact. If $K(X)$ denotes the constant functions on $X$ and if we now take $V=K^{+}(X)$, then $C V_{0}(X)$ is $\left(C_{0}(X),\|\cdot\|\right)$. One of the more important examples of a weighted space (and the space which motivated our interest in weighted spaces) is $C_{b}(X)$ with the strict topology $\beta$ (see Buck [2]), where $X$ is assumed to be locally compact. In fact, it follows easily from a result due to Buck [2, p. 97] that when $X$ is locally compact and $V=C_{0}^{+}(X)$, then $C V_{0}(X)=\left(C_{b}(X), \beta\right)$.

We now consider the question of the completeness of $C V_{0}(X)$. The difficulty arose in finding the proper setting for our general situation so as to include a sufficiently broad spectrum of weighted spaces. It is here that our partial order on Nachbin families plays a significant role, since our answer (given in 3.6 and 3.7) might properly be termed a "comparison test" for completeness.

3.5. Lemma. $N(X) \cap B_{0}(X)$ is complete in the uniform topology.

Proof. It will suffice to show $N(X) \cap B_{0}(X)$ is closed in $B(X)$. To do this, let $f \in B(X)$ be a limit point of $N(X) \cap B_{0}(X)$ in $B(X)$, let $\varepsilon>0$, and let

$$
F=\{x \in X:|f(x)| \geqq \varepsilon\} .
$$

Fix $x_{0} \in X \backslash F$, let $\eta=\frac{1}{2}\left(\varepsilon-\left|f\left(x_{0}\right)\right|\right)$, and choose $g \in N(X) \cap B_{0}(X)$ so that $\|g-f\|<\eta$. Then $A=\left\{x \in X:|g(x)|<\frac{1}{2}\left(\varepsilon+\left|f\left(x_{0}\right)\right|\right)\right\}$ is open, $x_{0} \in A$, and $A \subseteq X \backslash F$. Hence $F$ is closed, and this implies $f \in N(X)$. Now choose $h \in N(X) \cap B_{0}(X)$ so that $\|h-f\|$ $<\varepsilon / 2$, and let $K=\{x \in X:|h(x)| \geqq \varepsilon / 2\}$. Thus $K$ is compact, while $F \subseteq K$ implies $F$ is compact.

3.6 THEOREM. Let $U$ be a Nachbin family on $X$ for which the following properties hold:

(1) if $x \in X$, then there is $a u \in U$ so that $u(x)>0$;

(2) $C U_{0}(X)$ is complete.

If $V$ is a Nachbin family on $X$ with $U \leqq V$, then $C V_{0}(X)$ is complete. 
Proof. Let $\left\{f_{i}\right\}$ be an $\omega_{V}$-Cauchy net in $C V_{0}(X)$. It follows from 3.1 that $\left\{f_{i}\right\}$ is $\omega_{U}$-Cauchy, and hence there exists $f \in C(X)$ so that $f_{i} \rightarrow f\left(\omega_{U}\right)$. For $v \in V,\left\{f_{i} v\right\}$ is a uniformly Cauchy net in $N(X) \cap B_{0}(X)$, and hence by 3.5 there exists $f_{v} \in N(X)$ $\cap B_{0}(X)$ so that $f_{i} v \rightarrow f_{v}$ uniformly. From (1), $f v=f_{v}$ for every $v \in V$, and this implies $f \in C V_{0}(X)$, while it is clear that $f_{i} \rightarrow f\left(\omega_{V}\right)$.

Since $(C(X), \mathrm{c}-\mathrm{op})$ is complete whenever $X$ is a $k$-space [11, p. 267], we have the following result.

3.7. Corollary. If $X$ is a $k$-space and if $V$ is a Nachbin family on $X$ with $\chi_{c}(X) \leqq V$, then $C V_{0}(X)$ is complete.

We now turn our attention to the characterization of the topological dual space $C V_{0}(X)^{*}$ of $C V_{0}(X)$. The result given below (see Theorem 3.9), though adequate for the application intended in this paper (see Theorem 4.3), actually holds in a more general setting (see [10]), in which case the proof is considerably more complicated.

For the remainder of this section we will assume $X$ is locally compact. $M(X)$ will denote the complex Radon measures on $X$, while $M_{b}(X)$ will denote the subspace of bounded Radon measures. Define $V \cdot M_{b}(X)=\left\{v \cdot \mu: v \in V, \mu \in M_{b}(X)\right\}$ where $V$ is a Nachbin family on $X$ and $v \cdot \mu$ is the Radon measure on $X$ defined by $v \cdot \mu(f)=\mu(v f), f \in C_{c}(X)$. The technique employed in the lemma below was first used by Conway [5] in his characterization of the $\beta$-equicontinuous subsets of $M_{b}(X)$.

3.8. Lemma. Let $X$ be locally compact, let $V$ be a Nachbin family on $X$ with $V \subseteq C^{+}(X)$, and define $T_{v}: C V_{0}(X) \rightarrow C_{0}(X)$ for each $v \in V$ by $T_{v}(f)=f v$. If $v \in V$, then $V_{v}^{0}=T_{v}^{*}\left(B^{0}\right)$ where $B=\left\{f \in C_{0}(X):\|f\| \leqq 1\right\}$.

Proof. $T_{v}$ is clearly a well-defined linear and continuous map into $C_{0}(X)$, and hence $T_{v}^{*}$ is a weak-* continuous linear map of $M_{b}(X)$ into $C V_{0}(X)^{*}$. Now $B^{0}$ is $\sigma\left(M_{b}(X), C_{0}(X)\right)$-compact by Alaoglu's theorem, and so $T_{v}^{*}\left(B^{0}\right)$ is $\sigma\left(C V_{0}(X)^{*}, C V_{0}(X)\right)$-compact, while $T_{v}^{*}\left(B^{0}\right)$ is obviously absolutely convex. Since $V_{v}=T_{v}^{-1}(B)=\left\{T_{v}^{*}\left(B^{0}\right)\right\}^{0}$, it follows that $V_{v}^{0}=\left\{T_{v}^{*}\left(B^{0}\right)\right\}^{00}=T_{v}^{*}\left(B^{0}\right)$.

3.9. THEOREM. Let $X$ be locally compact and let $V$ be a Nachbin family on $X$ with $V \subseteq C^{+}(X)$. Then $V \cdot M_{b}(X)$ is a linear subspace of $M(X)$ and $T: V \cdot M_{b}(X) \rightarrow$ $C V_{0}(X)^{*}$ is a (linear) onto isomorphism where $T(v \cdot \mu)(f)=\int f v d \mu$ for each $f \in C V_{0}(X)$. In this case we will write $C V_{0}(X)^{*}=V \cdot M_{b}(X)$.

Proof. From the fact that $C_{c}(X)$ is $\omega$-dense in $C V_{0}(X)[9$, p. 64], one routinely verifies that $T$ is a well-defined function into $C V_{0}(X)^{*}$ and that $T$ is one-to-one. We now show that $T$ is onto, and, to this end, fix $L \in C V_{0}(X)^{*}$. It now follows from 3.8 that there exists $v \in V, \mu \in M_{b}(X)$ such that $L=T_{v}^{*} \mu$. If $f \in C V_{0}(X)$, then $\langle f, L\rangle=\left\langle f, T_{v}^{*} \mu\right\rangle=\left\langle T_{v} f, \mu\right\rangle=\langle f v, \mu\rangle=\int f v d \mu=\langle f, T(v \cdot \mu)\rangle$, from which it follows 
that $L=T(v \cdot \mu)$. It is now easy to see that $V \cdot M_{b}(X)$ is a linear subspace of $M(X)$ and that $T$ is indeed a linear onto isomorphism.

3.10. Corollary (Buck $\left[2\right.$, p. 99]). Let $X$ be locally compact. Then $\left(C_{b}(X), \beta\right)^{*}$ is $M_{b}(X)$.

Proof. As was pointed out in [3], a direct application of Hewitt's factorization theorem [7, p. 151] yields $C_{0}^{+}(X) \cdot M_{b}(X)=M_{b}(X)$. This fact together with 3.9 yield the desired result (and an alternate proof of Buck's theorem).

We now consider the problem of characterizing the extremal points $\mathscr{E}\left(V_{v}^{0}\right)$ of $V_{v}^{0}$ where $V$ is a Nachbin family on $X$ with $V \subseteq C^{+}(X)$ and $v \in V$. Here again, the technique is essentially that used by Conway [4] to obtain this result in the special case when $V=C_{0}^{+}(X)$. As yet, we have not been able to remove the continuity assumption on $V$ although we conjecture the characterization would be the same if, for example, $C_{c}^{+}(X) \leqq V \leqq C_{0}^{+}(X)$. In view of the forthcoming application of the following theorem, however, we feel it would be desirable to remove the continuity assumption.

3.11. Lemma. Let $X$ be locally compact, let $V$ be a Nachbin family on $X$ with $V \subseteq C^{+}(X)$, let $B=\left\{f \in C_{0}(X):\|f\| \leqq 1\right\}$, and let $\mu \in B^{0}$. If $v \in V$ and if $T_{v}^{*} \mu=T_{v}^{*} \delta(x)$ where $\delta(x)$ denotes the point mass at $x \in N(v)$ and $T_{v}$ is the map defined in 3.8 , then $\mu=\delta(x)$.

Proof. Let $\nu=\mu-\delta(x)$ and let $\phi \in C_{c}^{+}(X)$ with spt $(\phi) \subseteq N(v)$. Then $\phi_{v} \in C_{c}^{+}(X)$ and $\phi_{v} v=\phi$ where

$$
\begin{aligned}
\phi_{v}(y) & =\phi(y) / v(y), & & y \in N(v), \\
& =0, & & y \in X \mid \operatorname{spt}(\phi) .
\end{aligned}
$$

Since $T_{v}^{*} \nu=0, \int \phi d \nu=\int \phi_{v} v d \nu=\int \phi_{v} d\left(T_{v}^{*} \nu\right)=0$, and thus $|\nu|(N(v))=0$. Therefore $\mu(\{x\})=\nu(\{x\})+1=1$. If $A$ is any Borel set in $X$ and $x \notin A$, then

$$
1 \leqq|\mu|(A)+1=|\mu|(A \cup\{x\}) \leqq 1,
$$

which implies $|\mu|(A)=0$; i.e., $\mu=\delta(x)$.

In the proof of the following theorem we will need an extension of a result of Arens and Kelley which is due to Conway [4]. In particular, Conway showed that

$$
\mathscr{E}\left(B^{0}\right)=\{\lambda \delta(x):|\lambda|=1, x \in X\}
$$

where the notation is that of Lemma 3.11. Since this result is unpublished, we will give a brief indication of Conway's argument. To this end, take $\mu \in \mathscr{E}\left(B^{\circ}\right)$, let

$$
\mathscr{K}=\{K \subseteq X: K \text { is compact and }|\mu|(K)>0\},
$$

and note that $\mathscr{K}$ is nonvoid. If $K_{1}, K_{2} \in \mathscr{K}$ and if $K_{3}=X \mid\left(K_{1} \cup K_{2}\right)$, then $\mu=$ $\sum_{i=1}^{3}|\mu|\left(K_{t}\right) \mu_{i}$ where for any Borel set $A$ we define $\mu_{i}(A)=|\mu|\left(K_{i}\right)^{-1} \mu\left(A \cap K_{i}\right)$ if $|\mu|\left(K_{i}\right) \neq 0$ and zero otherwise. It readily follows that $\mu=\mu_{1}$ from whence $K_{1} \cap K_{2}$ is not void, and this together with the inner regularity of $\mu$ imply $K_{1} \cap K_{2} \in \mathscr{K}$. Consequently, there is an $x \in \bigcap\{K: K \in \mathscr{K}\}$ and $\mu=\mu(X) \delta(x)$. 
3.12. THEOREM. If $X$ is locally compact and if $V$ is a Nachbin family on $X$ with $V \subseteq C^{+}(X)$, then $\mathscr{E}\left(V_{v}^{0}\right)=\{\lambda v(x) \delta(x): x \in N(v),|\lambda|=1\}$, for every $v \in V$.

Proof. Fix $v \in V$ and let $T_{v}: C V_{0}(X) \rightarrow C_{0}(X)$ be the map defined in 3.8. If $B=\left\{f \in C_{0}(X): \| \leqq 1\right\}$, then $R\left(T_{v}^{*} ; B^{0}\right)$ is affine, while $T_{v}^{*}\left(B^{0}\right)=V_{v}^{0}$ by 3.8. Therefore, we have that $\mu \in \mathscr{E}\left(V_{v}^{0}\right)$ implies there is a $\nu \in \mathscr{E}\left(B^{0}\right)$ so that $\mu=T_{v}^{*} \nu=v \cdot \nu$. As was shown above, $\nu \in \mathscr{E}\left(B^{0}\right)$ implies there exist $x \in X, \lambda \in C,|\lambda|=1$, such that $\nu=\lambda \delta(x)$. Thus $\mu=\lambda v \cdot \delta(x)=\lambda v(x) \delta(x)$, and since $\mu \neq 0, x \in N(v)$.

Now let $x \in N(v)$ and let $\mu=v(x) \delta(x)$. Clearly, $\mu \in V_{v}^{0}$, and if $\mu=\frac{1}{2}(\sigma+\tau)$ where $\sigma, \tau \in V_{v}^{0}$, then there are $\alpha, \beta \in B^{0}$ such that $T_{v}^{*} \alpha=\sigma$ and $T_{v}^{*} \beta=\tau$. Thus $\mu=T_{v}^{*} \delta(x)$ $=T_{v}^{*}\left(\frac{1}{2}(\alpha+\beta)\right)$, and by $3.11, \delta(x)=\frac{1}{2}(\alpha+\beta)$. However, $\delta(x) \in \mathscr{E}\left(B^{0}\right)$, which implies $\delta(x)=\alpha=\beta$, and hence $\mu=\sigma=\tau$; i.e., $\mu \in \mathscr{E}\left(V_{v}^{0}\right)$.

4. Tensor products of weighted spaces. In this section we bring to bear the material developed in the preceding sections, which, in conjunction with Nachbin's important Weighted Dieudonné Density Theorem [9], gives us the representation theorem promised in $\$ 1$ (see Theorem 4.3, below).

If $f$ is a function on $X$ and if $g$ is a function on $Y$, then $f \times g$ will denote the function on $X \times Y$ defined by $f \times g(x, y)=f(x) g(y)$. In particular, if $U$ is a Nachbin family on $X$ and $V$ is a Nachbin family on $Y$, then $U \times V=W=\{u \times v: u \in U, v \in V\}$ is a Nachbin family on $X \times Y$ for which $C W_{0}(X \times Y)$ is Hausdorff whenever $Y$ (or $X$ ) is locally compact (recall our assumption that $C U_{0}(X)$ and $C V_{0}(Y)$ are Hausdorff). If we define $T: C(X) \otimes C(Y) \rightarrow C(X \times Y)$ by $T(f \otimes g)=f \times g$ and then extending linearly, $T$ is a one-to-one linear map, which will be referred to as the canonical embedding map.

Nachbin, in his book [9] which appeared in print this year, had considered part of the problem to which we have addressed ourselves, and we will use his Weighted Dieudonné Density Theorem (see 4.1, below) to replace our own somewhat weaker version of this result. This allows us to give an improved version of our Theorem 4.3.

4.1. Lemma (Weighted Dieudonné Density Theorem [9, p. 68]). If $U$ is a Nachbin family on $X$, if $V$ is a Nachbin family on $Y$, if $W=U \times V$, and if $T$ is the canonical embedding map, then $T$ maps $C U_{0}(X) \otimes C V_{0}(Y)$ into $C W_{0}(X \times Y)$ and $T\left(C U_{0}(X) \otimes C V_{0}(Y)\right)$ is $\omega_{W}$-dense in $C W_{0}(X \times Y)$.

We now state and prove the lemma which, together with 4.1 , constitutes the basis of the proof of 4.3. It is here that we apply the material of the preceding sections.

4.2. Lemma. Let $X$ and $Y$ be locally compact, let $U$ be a Nachbin family on $X$ with $U \subseteq C^{+}(X)$, let $V$ be a Nachbin family on $Y$ with $V \subseteq C^{+}(Y)$, and let $W=U \times V$. If $T$ is the canonical embedding map, then $T$ establishes a topological isomorphism between $C U_{0}(X) \otimes C V_{0}(Y)$ endowed with the biequicontinuous topology and $T\left(C U_{0}(X) \otimes C V_{0}(Y)\right)$ endowed with the relative $\omega_{W}$-topology. 
Proof. $T$ is a linear isomorphism which maps $C U_{0}(X) \otimes C V_{0}(Y)$ into $C W_{0}(X \times Y)$ by 4.1 . It suffices then to show $T$ is bicontinuous. To do this, let $F \in C U_{0}(X) \otimes C V_{0}(Y)$, let $u \in U$, and let $v \in V$; there exists $\left\{f_{k}\right\}_{k=1}^{n} \subseteq C U_{0}(X)$, $\left\{g_{k}\right\}_{k=1}^{n} \subseteq C V_{0}(Y)$ such that $F=\sum_{k=1}^{n} f_{k} \otimes g_{k}$. For $y \in N(v), h_{y}=\sum_{k=1}^{n} g_{k}(y) v(y) f_{k}$ is in $C U_{0}(X)$, and it follows from the Krein-Milman theorem that there is a $\mu \in \mathscr{E}\left(V_{u}^{0}\right)$ such that $\left|\left\langle h_{y}, \mu\right\rangle\right|=\sup \left\{\left|\left\langle h_{y}, \nu\right\rangle\right|: \nu \in V_{u}^{0}\right\}$. Similarly, for $\mu \in V_{u}^{0}$, $G_{\mu}=\sum_{k=1}^{n}\left\langle f_{k}, \mu\right\rangle g_{k}$ is in $C V_{0}(Y)$, which implies there is a $\nu \in \mathscr{E}\left(V_{v}^{0}\right)$ so that $\left|\left\langle G_{\mu}, \nu\right\rangle\right|=\sup \left\{\left|\left\langle G_{u}, \sigma\right\rangle\right|: \sigma \in V_{v}^{0}\right\}$. By 3.12, $\mu \in \mathscr{E}\left(V_{u}^{0}\right), \nu \in \mathscr{E}\left(V_{v}^{0}\right)$ if and only if there exists $x \in N(u), y \in N(v)$ and $\alpha, \beta \in C$ with $|\alpha|=|\beta|=1$ such that $\mu=\alpha u(x) \delta(x)$, $\nu=\beta v(y) \delta(y)$. Therefore for $w=u \times v$ we have

$$
\begin{aligned}
\|T(F) w\| & =\sup \left\{\left|\sum_{k=1}^{n} f_{k}(x) g_{k}(y) u(x) v(y)\right|: x \in N(u), y \in N(v)\right\} \\
& =\sup \left\{\left|h_{y}(x) u(x)\right|: x \in N(u), y \in N(v)\right\} \\
& =\sup \left\{\left|\left\langle h_{y}, u(x) \delta(x)\right\rangle\right|: x \in N(u), y \in N(v)\right\} \\
& =\sup \left\{\left|\left\langle h_{y}, \mu\right\rangle\right|: \mu \in V_{u}^{0}, y \in N(v)\right\} \\
& =\sup \left\{\left|G_{\mu}(y) v(y)\right|: \mu \in V_{u}^{0}, y \in N(v)\right\} \\
& =\sup \left\{\left|\left\langle G_{u}, v(y) \delta(y)\right\rangle\right|: \mu \in V_{u}^{0}, y \in N(v)\right\} \\
& =\sup \left\{\left|\left\langle G_{u}, v\right\rangle\right|: \mu \in V_{u}^{0}, \nu \in V_{v}^{0}\right\} \\
& =\sup \left\{\left|\sum_{k=1}^{n}\left\langle f_{k}, \mu\right\rangle\left\langle g_{k}, \nu\right\rangle\right|: \mu \in V_{u}^{0}, \nu \in V_{v}^{0}\right\} \\
& =\sup \left\{|F(\mu, \nu)|: \mu \in V_{u}^{0}, \nu \in V_{v}^{0}\right\},
\end{aligned}
$$

and the proof is complete.

4.3. THEOREM. Let $X$ and $Y$ be locally compact, let $U$ be a Nachbin family on $X$ with $C_{c}^{+}(X) \leqq U \subseteq C^{+}(X)$, let $V$ be a Nachbin family on $Y$ with $C_{c}^{+}(Y) \leqq V \subseteq C^{+}(Y)$, and let $W=U \times V$. Then $C U_{0}(X) \otimes \approx C V_{0}(Y)$ is topologically isomorphic to $C W_{0}(X \times Y)$.

Proof. $T\left(C U_{0}(X) \otimes C V_{0}(Y)\right)$ is $\omega_{W}$-dense in $C W_{0}(X \times Y)$ by 4.1 , while the local compactness of $X$ and $Y$ implies $C_{c}^{+}(X \times Y) \leqq W$, and hence $C W_{0}(X \times Y)$ is complete by 3.7. The result is now immediate from 4.2 .

4.4. Corollary (Grothendieck [6, I, p. 90]). If $X$ and $Y$ are locally compact, then $\left(C_{0}(X),\|\cdot\|\right) \otimes \approx\left(C_{0}(Y),\|\cdot\|\right)$ is topologically isomorphic to $\left(C_{0}(X \times Y),\|\cdot\|\right)$.

Proof. If $U=K^{+}(X), V=K^{+}(Y)$, then the hypothesis of 4.3 is satisfied. Since $C U_{0}(X)=\left(C_{0}(X),\|\cdot\|\right), C V_{0}(Y)=\left(C_{0}(Y),\|\cdot\|\right)$, it will suffice, in view of 3.2 , to show $W \approx K^{+}(X \times Y)$ where $W=U \times V$, and this is clear.

4.5. COROLLARY. If $X$ and $Y$ are locally compact, then

$$
(C(X), c-o p) \otimes \approx(C(Y), c-o p)
$$

is topologically isomorphic to $(C(X \times Y), c-o p)$. 
Proof. Take $U=C_{c}^{+}(X), V=C_{c}^{+}(Y)$, and $W=U \times V$. Then the hypothesis of 4.3 is satisfied, while it is clear that $W \approx C_{c}^{+}(X \times Y)$. The result now follows from 3.4.

4.6. LemMA. If $X$ and $Y$ are locally compact, if $U=C_{0}^{+}(X)$ and $V=C_{0}^{+}(Y)$, and if $W=U \times V$, then $W \approx C_{0}^{+}(X \times Y)$.

Proof. If $u \in U, v \in V$, then $w \in C^{+}(X \times Y)$ where $w=u \times v$. Taking $\varepsilon>0$ and assuming $u, v \neq 0, A=\{(x, y): w(x, y) \geqq \varepsilon\}$ is closed, and $A \subseteq\left\{x \in X: u(x) \geqq \varepsilon\|v\|^{-1}\right\}$ $\times\left\{y \in Y: v(y) \geqq \varepsilon\|u\|^{-1}\right\}$ from whence it follows that $A$ is compact. Therefore $W \subseteq C_{0}^{+}(X \times Y)$.

Now let $\phi \in C_{0}^{+}(X \times Y)$, and assume $\|\phi\| \leqq 1$. For each $n$, let

$$
K_{n}=\left\{(x, y): 4^{-n} \leqq \phi(x, y) \leqq 4^{-(n-1)}\right\},
$$

let $F_{n}=\Pi_{X}\left(K_{n}\right)$, and let $G_{n}=\Pi_{Y}\left(K_{n}\right)$. Hence for every $n$ there exists $u_{n} \in C_{c}(X)$, $v_{n} \in C_{c}(Y)$ so that $0 \leqq u_{n}, v_{n} \leqq 1$ and $u_{n}\left(F_{n}\right)=v_{n}\left(G_{n}\right)=1$. Define $u=\sum_{n=1}^{\infty} 2^{-(n-1)} u_{n}$, and observe that $u \in C_{0}^{+}(X)$, while $v=\sum_{n=1}^{\infty} 2^{-(n-1)} v_{n}$ is in $C_{0}^{+}(Y)$. If $(x, y) \in N(\phi)$, then there is an $n$ such that $(x, y) \in K_{n}$. Thus $u(x) \geqq 2^{-(n-1)}$ and $v(y) \geqq 2^{-(n-1)}$, which implies $u \times v(x, y) \geqq 4^{-(n-1)} \geqq \phi(x, y)$; i.e., $C_{0}^{+}(X \times Y) \leqq W$.

4.7. THEOREM. If $X$ and $Y$ are locally compact, then $\left(C_{b}(X), \beta\right) \otimes \approx\left(C_{b}(Y), \beta\right)$ is topologically isomorphic to $\left(C_{b}(X \times Y), \beta\right)$.

Proof. The hypothesis of 4.3 is satisfied for $U=C_{0}^{+}(X)$ and $V=C_{0}^{+}(Y)$. Since 4.6 shows that $W \approx C_{0}^{+}(X \times Y)$ where $W=U \times V$, the result follows easily in view of 3.4 .

5. Subspaces of $C(X \times Y)$. In addition to the preceding applications of 4.3, we are able to apply it to other combinations of $U$ and $V$ to obtain certain new subspaces of $C(X \times Y)$. Moreover, in view of our characterization of $C V_{0}(X)^{*}$ (Theorem 3.9), 4.3 yields a simple characterization of $\left[C U_{0}(X) \otimes \approx C V_{0}(Y)\right]^{*}$ whenever it applies (for example, compare Grothendieck [6, I, p. 124]).

A function $f$ on $X \times Y$ will be called compact column bounded if $R(f ; K \times Y)$ $\in C_{b}(K \times Y)$ for every compact subset $K$ of $X$. Note that a compact column bounded function $f$ is in $C(X \times Y)$ whenever $X$ is locally compact. We will denote by $C C_{b}(X \times Y)$ the set of all $f \in C(X \times Y)$ such that $f$ is compact column bounded. Clearly, $C C_{b}(X \times Y)$ is a subalgebra of $C(X \times Y)$ and $C_{b}(X \times Y) \subseteq C C_{b}(X \times Y)$ $\subseteq C(X \times Y)$, where both containments can be proper. If $X$ is locally compact, then a function $f$ on $X \times Y$ is in $C C_{b}(X \times Y)$ if and only if $f(\phi \times 1) \in C_{b}(X \times Y)$ for every $\phi \in C_{c}^{+}(X)$.

5.1. THEOREM. If $X$ and $Y$ are locally compact, then $(C(X), c-o p) \otimes \approx\left(C_{b}(Y), \beta\right)$ is topologically isomorphic to $C W_{0}(X \times Y)$ where $W=C_{c}^{+}(X) \times C_{0}^{+}(Y)$ and

$$
C W_{0}(X \times Y)=C C_{b}(X \times Y)
$$

(as sets). 
For the proof of 5.1, we make use of the following lemma.

5.2. Lemma. Let $X$ be compact and let $\phi \in C(X \times Y)$. If

$$
\theta(y)=\sup \{|\phi(x, y)|: x \in X\}
$$

then $\theta \in C^{+}(Y)$. Moreover, if $\phi \in C_{b}(X \times Y)$, then $\theta \in C_{b}^{+}(Y)$; if $\phi \in C_{0}(X \times Y)$, then $\theta \in C_{0}^{+}(Y)$; and if $\phi \in C_{c}(X \times Y)$, then $\theta \in C_{c}^{+}(Y)$.

Proof. $\theta$ is obviously well defined and $\theta \geqq 0$. Fix $y_{0} \in Y$ and let $\varepsilon>0$. For each $x \in X$ there is an open neighborhood $A(x)$ of $x$ and an open neighborhood $B(x)$ of $y_{0}$ such that $(t, y) \in A(x) \times B(x)$ implies $\left\|\phi(t, y)|-| \phi\left(x, y_{0}\right)\right\|<\varepsilon / 8$. Since $X \times\left\{y_{0}\right\}$ is compact, there exists $\left\{x_{k}\right\}_{k=1}^{n} \subseteq X$ such that $\left\{A\left(x_{k}\right) \times B\left(x_{k}\right)\right\}_{k=1}^{n}$ covers $X \times\left\{y_{0}\right\}$. Let $B=\bigcap_{k=1}^{n} B\left(x_{k}\right)$ and let $y \in B$. Now $B$ is an open neighborhood of $y_{0}$, and if $\left(x, y_{0}\right) \in A\left(x_{k}\right) \times B\left(x_{k}\right)$ for some $k \in\{1, \ldots, n\}$, then $(x, y) \in A\left(x_{k}\right) \times B\left(x_{k}\right)$. Also observe that, since $X$ is compact, for every $p \in Y$ there is an $x_{p} \in X$ so that $\theta(p)=\left|\phi\left(x_{p}, p\right)\right|$. If $\left|\theta\left(y_{0}\right)-\right| \phi\left(x_{y}, y_{0}\right)||>\varepsilon / 2$, then, since there exist $k_{1}$ and $k_{2}$ such that $\left(x_{y}, y_{0}\right) \in A\left(x_{k_{1}}\right) \times B\left(x_{k_{1}}\right)$ and $\left(x_{y_{0}}, y_{0}\right) \in A\left(x_{k_{2}}\right) \times B\left(x_{k_{2}}\right)$,

$$
\begin{aligned}
0 \leqq \theta(y)-\left|\phi\left(x_{y_{0}}, y\right)\right| & =\theta(y)-\left|\phi\left(x_{y}, y_{0}\right)\right|+\left|\phi\left(x_{y}, y_{0}\right)\right|-\theta\left(y_{0}\right)+\theta\left(y_{0}\right)-\left|\phi\left(x_{y_{0}}, y\right)\right| \\
& <\varepsilon / 4+(-\varepsilon / 2)+\varepsilon / 4=0,
\end{aligned}
$$

which is impossible. Hence $\left|\theta\left(y_{0}\right)-\right| \phi\left(x_{y}, y_{0}\right)|| \leqq \varepsilon / 2$ and

$$
\left|\theta\left(y_{0}\right)-\theta(y)\right| \leqq\left|\theta\left(y_{0}\right)-\right| \phi\left(x_{y}, y_{0}\right)||+|| \phi\left(x_{y}, y_{0}\right)|-\theta(y)|<\varepsilon / 2+\varepsilon / 4<\varepsilon,
$$

from which it follows that $\theta \in C^{+}(Y)$.

Now if $\phi \in C_{b}(X \times Y)$, then $\theta(y)=\left|\phi\left(x_{y}, y\right)\right| \leqq\|\phi\|$ for every $y \in Y$; i.e., $\theta \in C_{b}^{+}(Y)$. Moreover, if $\phi \in C_{c}(X \times Y)$ and if $y \in N(\theta)$, then $0<\theta(y)=\left|\phi\left(x_{y}, y\right)\right|$. This implies $\left(x_{y}, y\right) \in N(\phi)$ and therefore $y \in \Pi_{Y}\left(N(\phi)^{-}\right)$, which is compact. Hence spt $(\theta)$ is compact and $\theta \in C_{c}^{+}(Y)$. Now assume $\phi \in C_{0}(X \times Y)$, let $\varepsilon>0$, and let

$$
F=\{y \in Y: \theta(y) \geqq \varepsilon\} .
$$

If $y \in F$, then $\varepsilon \leqq \theta(y)=\left|\phi\left(x_{y}, y\right)\right|$, which implies $y \in \Pi_{Y}\{(x, y):|\phi(x, y)| \geqq \varepsilon\}$. Since $F$ is closed and contained in a compact set, $F$ is compact, and from this we have $\theta \in C_{0}^{+}(Y)$.

Proof of 5.1. In view of 4.3, it will suffice to show $C W_{0}(X \times Y)=C C_{b}(X \times Y)$. To this end, assume that $f \in C C_{b}(X \times Y)$, let $u \in U, v \in V$, let $w=u \times v$, and choose $\phi \in C_{c}^{+}(X)$ so that $\phi($ spt $(u))=1$. From the proof of $4.6, \phi \times v \in C_{0}^{+}(X \times Y)$, while $f(u \times 1) \in C_{b}(X \times Y)$. Since $f w=f(u \times 1)(\phi \times v)$, we have $f w \in C_{0}(X \times Y)$; i.e., $f \in C W_{0}(X \times Y)$.

Now assume $f \in C W_{0}(X \times Y)$, fix $\phi \in C_{c}^{+}(X)$, and let $\psi \in C_{0}(X \times Y)$. Since $R(\psi ; \operatorname{spt}(\phi) \times Y) \in C_{0}(\operatorname{spt}(\phi) \times Y)$, we have from 5.2 that $\theta \in C_{0}^{+}(Y)$ where 
$\theta(y)=\sup \{|R(\psi: \operatorname{spt}(\phi) \times Y)(x, y)|: x \in \operatorname{spt}(\phi)\}$. Therefore $\phi \times \theta \in W$, which implies $f(\phi \times \theta) \in C_{0}(X \times Y)$. Choose $\varepsilon>0$ and let

$$
A=\{(x, y):|f(x, y) \psi(x, y)|(\phi \times 1)(x, y) \geqq \varepsilon\} .
$$

Now $A \subseteq\{(x, y):|f(x, y)|(\phi \times \theta)(x, y) \geqq \varepsilon\}$, and hence $A$ is compact. But this says $f(\phi \times 1) \psi=C_{0}(X \times Y)$, and so $f(\phi \times 1) \in C_{b}(X \times Y)$ [2, p. 97], and it now follows that $f \in C C_{b}(X \times Y)$.

A function $f$ on $X \times Y$ is called compact column vanishing at infinity if

$$
R(f ; K \times Y) \in C_{0}(K \times Y)
$$

for every compact subset $K$ of $X$. We denote the set of all $f \in C(X \times Y)$ such that $f$ is compact column vanishing at infinity by $C C_{0}(X \times Y) . C C_{0}(X \times Y)$ is a subalgebra of $C(X \times Y)$, and $C_{0}(X \times Y) \subseteq C C_{0}(X \times Y) \subseteq C C_{b}(X \times Y)$ where both inclusions can be proper. If $X$ is locally compact, then a function $f$ on $X \times Y$ is in $C C_{0}(X \times Y)$ if and only if $f(\phi \times 1) \in C_{0}(X \times Y)$ for every $\phi \in C_{c}^{+}(X)$.

5.3. THEOREM. If $X$ and $Y$ are locally compact, then $(C(X), c-o p) \otimes \approx\left(C_{0}(Y),\|\cdot\|\right)$ is topologically isomorphic to $C W_{0}(X \times Y)$ where $W=C_{c}^{+}(X) \times K^{+}(Y)$ and

(as sets).

$$
C W_{0}(X \times Y)=C C_{0}(X \times Y)
$$

Proof. In view of 4.3, it suffices to show $C W_{0}(X \times Y)=C C_{0}(X \times Y)$. To do this, first assume $f \in C C_{0}(X \times Y)$, let $u \in U, v \in V$, and let $w=u \times v$. Since $v f \in C C_{0}(X \times Y)$, $f w=v f(u \times 1) \in C_{0}(X \times Y)$; i.e., $f \in C W_{0}(X \times Y)$.

If $f \in C W_{0}(X \times Y)$ and if $\phi \in C_{c}^{+}(X)$, then $f(\phi \times 1) \in C_{0}(X \times Y)$, and this implies $f \in C C_{0}(X \times Y)$.

5.4. THEOREM. If $X$ and $Y$ are locally compact, then $\left(C_{b}(X), \beta\right) \otimes \approx\left(C_{0}(Y),\|\cdot\|\right)$ is topologically isomorphic to $C W_{0}(X \times Y)$ where $W=C_{0}^{+}(X) \times K^{+}(Y)$ and $C W_{0}(X \times Y)=C C_{0}(X \times Y) \cap C_{b}(X \times Y)($ as sets $)$.

Proof. It will suffice to show $C W_{0}(X \times Y)=C C_{0}(X \times Y) \cap C_{b}(X \times Y)$, and, to this end, assume $f \in C C_{0}(X \times Y) \cap C_{b}(X \times Y)$, let $u \in U, v \in V$, and let $w=u \times v$. We may assume $f \neq 0, u \neq 0$, and $v \neq 0$, and in this case there is a $c>0$ such that $v(y)=c$ for each $y \in Y$. Choose $\varepsilon>0$ and let $A=\{(x, y):|f(x, y)| w(x, y) \geqq \varepsilon\}$. Now $A$ is closed and we wish to show $A$ is compact. Since $K=\left\{x \in X: u(x) \geqq \varepsilon(c\|f\|)^{-1}\right\}$ is compact, $B=\left\{(x, y) \in K \times Y:|f(x, y)| \geqq \varepsilon(c\|u\|)^{-1}\right\}$ is compact, and $A \subseteq B$.

Now assume $f \in C W_{0}(X \times Y)$. Consequently, for each $u \in C_{c}^{+}(X), f(u \times 1)$ $\in C_{0}(X \times Y)$, and so $f \in C C_{0}(X \times Y)$. By 4.6, $U \times C_{0}^{+}(Y) \approx C_{0}^{+}(X \times Y)$, while it is clear that $U \times C_{0}^{+}(Y) \leqq W$. Thus $C_{0}^{+}(X \times Y) \leqq W$, and 3.1 together with 3.4 now yield that $C W_{0}(X \times Y) \subseteq C_{b}(X \times Y)$.

We will conclude by summarizing the relationships between certain known spaces and the spaces introduced in this section in the following diagram, where each map 
is inclusion which is in general proper. It should be remarked at this point, that our "column" spaces give rise, of course, to analogous "row" spaces.

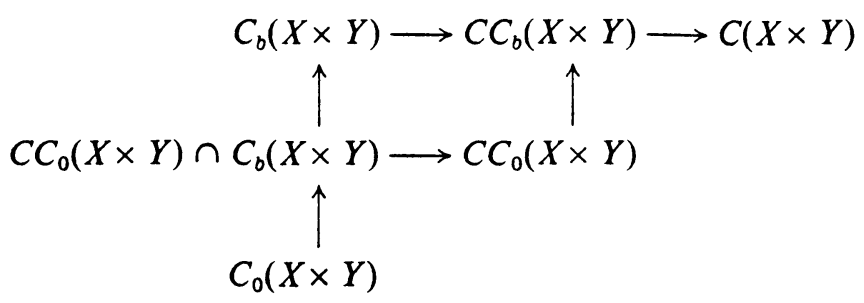

\section{REFERENCES}

1. S. Bernstein, Le problème de l'approximation des fonctions continues sur tout l'axe réel et l'une de ses applications, Bull. Soc. Math. France 52 (1924), 399-410.

2. R. C. Buck, Bounded continuous functions on a locally compact space, Michigan Math. J. 5 (1958), 95-104.

3. H. S. Collins and W. H. Summers, Some applications of Hewitt's factorization theorem, Proc. Amer. Math. Soc. 21 (1969), 727-733.

4. J. B. Conway, The strict topology and compactness in the space of measures, Ph.D. Dissertation, Louisiana State University, 1965.

5. —_ The strict topology and compactness in the space of measures, Bull. Amer. Math Soc. 72 (1966), 75-78.

6. A. Grothendieck, Produits tensoriels topologiques et espaces nucléaires, Mem. Amer. Math. Soc. No. 16, 1955.

7. E. Hewitt, The ranges of certain convolution operators, Math. Scand. 15 (1964), 147-155.

8. L. Nachbin, Weighted approximation for algebras and modules of continuous functions: real and self-adjoint complex cases, Ann. of Math. 81 (1965), 289-302.

9. - Elements of approximation theory, Van Nostrand, Princeton, N. J., 1967.

10. W. H. Summers, Weighted locally convex spaces of continuous functions, Ph.D. Dissertation, Louisiana State University, 1968.

11. S. Warner, The topology of compact convergence on continuous function spaces, Li!'e Math. J. 25 (1958), 265-282.

\section{Louisiana State University, \\ Baton Rouge, Louisiana \\ UNIVERSITY OF ARKANSAS, \\ FAYETTEVILle, ARKansas}

